\title{
Parámetros de crecimiento, mortalidad y tasa de explotación del pulpo Octopus maya en Holbox, Quintana Roo, México
}

Growth parameters, mortality and exploitation rate of Octopus maya, at Holbox, Quintana Roo, Mexico

\section{Aristeo Hernández-Sánchez ${ }^{1}$ y Alberto de Jesús-Navarrete ${ }^{2}$}

\author{
${ }^{1}$ Instituto Tecnológico de Chetumal, Departamento de Biología, Av. Insurgentes No 330, CP 77013. Chetumal, Quintana Roo, \\ México \\ ${ }^{2}$ Departamento de Aprovechamiento y Manejo de Recursos Acuáticos, El Colegio de la Frontera Sur, Unidad Chetumal, \\ Av. Centenario Km. 5.5 CP 77014. Chetumal, Quintana Roo, México. anavarre@ecosur.mx
}

\begin{abstract}
In the Yucatan Peninsula, southern Mexico, octopus fishery is based almost entirely on the exploitation of an endemic species, Octopus maya. In the state of Quintana Roo 0. maya is captured around the island of Holbox. To estimate some population parameters, monthly sampling of octopus captured by the smaller fleet at Holbox for commercialization were undertaken in the fishing seasons of 2002 and 2003. Differences in the composition of sexes were statistically significant, with a higher presence of females. Consequently, the evaluation of growth parameters using the von Bertalanffy equation was realized separately for females and males. For females, the parameters were: $L_{\infty}=215 \mathrm{~mm}$, of mantle length, $K=0.68$ year $^{-1} ; t_{0}=0.34$, while for males $L_{\infty}=240$ mm of mantle length, $K=0.99$ year $^{-1} ; t_{0}=0.35$. The length-weight relationship indicated an alometric relationship; $P=0.026 L^{2.16}$, for the females in 2002 and of $P=0.0076 L^{2.45}$ for the females in 2003. In the case of males the length-weight relationship was $P=0.015 L^{2.27}$ for 2002 , and of $P=0.038 L^{2.12}$ for 2003 , where $P$ is the wet weight in $g$ and $L$ the mantle length in $\mathrm{mm}$. Total mortality were: $\mathrm{Z}=3.16$ and $\mathrm{M}=1.49, \mathrm{~F}=1.67$. The preliminary evaluation of the exploitation rate was $\mathrm{E}=0.53$ which indicates that the resource is exploited adequately.
\end{abstract}

Key words: von Bertalanffy parameters, alometric relationship, total mortality, Yucatán Peninsula

Resumen.- En la península de Yucatán, sur de México, la pesquería de pulpo se basa en la explotación casi exclusiva de la especie endémica Octopus maya, en el estado de Quintana Roo este recurso se captura en Holbox. Para estimar algunos parámetros poblacionales, se realizaron muestreos mensuales de la captura comercial de la flota menor de Holbox en las temporadas de pesca 2002 y 2003. Las diferencias en la composición por sexos fueron estadísticamente significativas, con una mayor presencia de hembras. Por lo tanto, se procedió a evaluar los parámetros de crecimiento de la ecuación de Von Bertalanffy para hembras y machos por separado. Para las hembras, los parámetros fueron: $L_{\infty}=215 \mathrm{~mm}$, de longitud de manto, $\mathrm{K}=0,68$ al año-1; $\mathrm{t}_{0}=0,343$, mientras que para los machos $L_{\infty}=240 \mathrm{~mm}$ de longitud de manto, $K=0,99$ al año-1; $t_{0}=0,354$. La relación longitud-peso indicó una relación alométrica; $P=0,026 L^{2,16}$, para las hembras en 2002 y de $P=0,0076 L^{2,45}$ para las hembras en 2003 . En el caso de los machos la relación longitud peso fue de $P=0,015 L^{2,27}$ para 2002, y de $P=0,038 L^{2,12}$ para 2003, donde $P$ es el peso húmedo en $\mathrm{g}$ y $\mathrm{L}$ la longitud del manto en $\mathrm{mm}$. Los coeficientes promedio de mortalidad estimados fueron: $Z=3,16$ y $M=1,49, F=1,67$. La evaluación preliminar del grado de explotación del recurso fue $E=0,53$, es decir, el recurso se está aprovechando adecuadamente.

Palabras clave: parámetros de von Bertalanffy, relación alométrica, mortalidad total, Península de Yucatán

\section{INTRODUCCIÓN}

En la Península de Yucatán existen varias especies pesqueras con un alto valor comercial, dentro de los invertebrados están la langosta espinosa del Caribe (Panulirus argus), los pulpos (Octopus vulgaris y Octopus maya) y los camarones peneidos. Entre los peces se encuentran el mero (Epinephelus morio), los pargos (Lutjanus griseus y Lutjanus analis), el canané (Ocyurus chrysurus), el negrillo (Mycteroperca bonaci), la cabrilla
(Mycteroperca venenosa), y el boquinete (Lachnolaimus maximus) (Sosa-Cordero et al. 1991).

La pesquería de pulpo en Yucatán es una actividad que data de 1942 (Solís-Ramírez \& Chávez 1986), pero fue hasta 1970 cuando se inició en ese Estado una pesca a escala comercial, al capturarse 304 ton (Arreguín-Sánchez et al. 1987). Con el aumento de las capturas se 
incrementaron también los estudios sobre la dinámica poblacional del recurso en el Banco de Campeche (Seijo et al. 1987, Arreguín-Sánchez 1992a, b, Chávez 1998, Solís-Ramírez et al. 1999).

El crecimiento del pulpo Octopus maya fue evaluado por Solís-Ramírez \& Chávez (1986), utilizando métodos indirectos basados en la ecuación de crecimiento de von Bertalanffy, y encontraron que es una especie con crecimiento rápido $\left(\mathrm{K}=0,263 \mathrm{año}^{-1}\right)$ lo que significaría una longevidad de 18 meses, esto es un ciclo de vida corto. Otras evaluaciones pesqueras abordaron el problema utilizando datos de frecuencia longitud, como ArreguínSánchez et al. (1987). Estos métodos tienen la ventaja de emitir un resultado rápido a bajo costo, sin embargo, su empleo en organismos de tejido suave como los pulpos se ha criticado, ya que se pueden tener distintos grados de contracción muscular (Forsythe \& Van Heukelem 1987), aunque desde luego los resultados de frecuencia longitud deberían ser validados posteriormente con otras técnicas como marcaje-recaptura (Domain et al. 2000) o bien técnicas moleculares (Moltschaniwskyj 2004). Recientemente se ha investigado el crecimiento de la especie comparando distintos métodos en la misma región (Nepita-Villanueva \& Defeo 2001), y encontraron que la constante de crecimiento del pulpo varió entre 0,50 a 1,35 año ${ }^{-1}$, en tanto que en Chile, con $O$. tehuelchus, Storero et al. (2010) informaron que la constante de crecimiento varió entre 0,49 a 0,58 año-1.

En Yucatán se han realizado estudios sobre la taxonomía, biología y comercialización de las especies de pulpos (De la Peña 1957, Solís-Ramírez 1962). Fuentes et al. (1965) estudiaron algunos aspectos de la reproducción de O. vulgaris; en tanto que Solís-Ramírez (1967) presentó un informe sobre el estudio de los octópodos de México, y estudió la incubación parcial de $O$. maya en el laboratorio.

En Quintana Roo, hasta 1990 no existía una pesquería formal de pulpo, sin embargo, en 1971, luego de una marea roja, arribaron grandes cantidades de pulpo a Holbox, (Solís-Ramírez 1975), iniciándose así una pesquería artesanal en 1991 (Sosa-Cordero et al. 1991). A diferencia de Yucatán, en Quintana Roo no existen trabajos previos sobre el estado y la dinámica del recurso, y aunque las investigaciones previas se han realizado en estados vecinos como Yucatán y Campeche, los datos no son extrapolables. Por lo anterior, los objetivos de esta investigación fueron: 1) determinar las características de la captura, y 2) calcular los parámetros poblacionales del pulpo en Holbox, datos que permitirán establecer alternativas de manejo, en un recurso que se está explotando y que debe ser evaluado adecuadamente.

\section{Material y Métodos}

Área de estudio. Holbox se ubica en las coordenadas geográficas $21^{\circ} 31^{\prime} 24^{\prime \prime} \mathrm{N}$ y $87^{\circ} 22^{\prime} 42^{\prime \prime} \mathrm{W}$. Tiene una longitud de $1,5 \mathrm{~km}$ de ancho y $44 \mathrm{~km}$ de largo. Al norte colinda con el Golfo de México y el Mar Caribe, al sur se encuentra la Laguna de Yalahau, la cual separa a la isla de la parte continental (Fig. 1).

Se realizaron muestreos mensuales, de agosto a diciembre, del pulpo Octopus maya, provenientes de la captura comercial de la flota menor de la isla de Holbox, en las temporadas de pesca 2002 y 2003. El pulpo se encuentra en veda de enero a julio. De cada organismo se midió la longitud de manto (LM), con un vernier al milímetro más cercano. El peso se determinó en gramos con una báscula digital de 0,050 g de sensibilidad. Los datos se separaron por sexo (que se identifica por la presencia del brazo copulador en machos). Se calculó la proporción de hembras: machos y se realizó una prueba Chi-cuadrado para determinar si la proporción es diferente de 1:1 (Zar 1999), fueron excluidos del análisis los individuos en que no fue posible identificar el sexo.

Los datos de longitud de ambas temporadas, se agruparon en intervalos de $10 \mathrm{~mm}$, siguiendo las recomendaciones de Defeo et al. (1992), y con ello se elaboraron histogramas de frecuencias de tallas y por sexos. Para determinar los parámetros de crecimiento se utilizó la aplicación informática FISAT II, considerando las frecuencias de tallas por separado para las hembras y los machos, aplicando los métodos ELEFAN y el de Shepherd (Shepherd's Length Composition Analysis) (Gayanilo et al. 1989) considerando que los datos, siguen la ecuación de crecimiento de von Bertalanffy. De manera similar, para

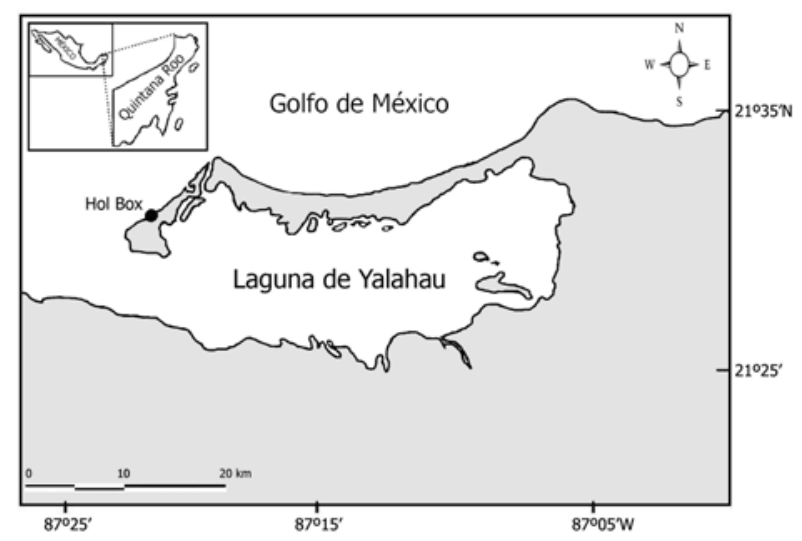

Figura 1. Localidad de muestreo, Holbox ubicado en la Península de Yucatán, México / Location of sampling, Holbox at Yucatan Peninsula, Mexico 
cada sexo se determinó la relación longitud-peso mediante una regresión potencial representada por la ecuación $\mathrm{P}=a \mathrm{~L}^{b}$, donde $\mathrm{a} \mathrm{y} \mathrm{b}$ son constantes de regresión. El valor de la pendiente $(b)$ y del intercepto $(a)$ para los machos y las hembras se compararon usando el análisis de covarianza (Zar 1999); la alometría de esta relación fue probada con la prueba t-Student para las hembras y los machos respectivamente. La mortalidad total (Z) se obtuvo de la curva de captura y la mortalidad natural (M) se calculó de acuerdo con la ecuación de Pauly (1987).

Se calculó la tasa de explotación utilizando la relación $\mathrm{E}=\mathrm{F} / \mathrm{Z}$, y suponiendo que el $\mathrm{E}_{\text {optimo }}$ ocurre cuando la mortalidad por pesca (F) es igual a la mortalidad natural $(\mathrm{M})$, por lo que de manera general se considera que $\mathrm{E}_{\text {optimo }}$ $=0,5$ (Gulland 1971).

\section{Resultados}

Se registró un total de 1.686 individuos en las temporadas de pesca de los años 2002 y 2003. En la temporada 2002, 744 individuos fueron registrados; 344 hembras y 181 machos. Otros 219 individuos fueron de sexo indefinido, los que no fueron considerados en este estudio. La talla promedio para las hembras fue de $129 \mathrm{~mm}( \pm 23,73) \mathrm{LM}$ y para machos fue de $130 \mathrm{~mm}( \pm 19,07)$. La proporción de sexos fue estadísticamente significativa entre hembras y machos (1,9: 1 respectivamente) $\left(\chi^{2}=352, P<0,05\right)$. En la temporada 2003, se obtuvieron 942 individuos; 765 hembras y 34 machos, y otros 143 individuos sexualmente indefinidos. Hubo mayor abundancia de hembras que de machos (22,02:1 respectivamente) $\left(\chi^{2}=178, P<0,05\right)$. La LM para las hembras fue de $125 \mathrm{~mm}( \pm 21,70)$ y para machos fue de $138 \mathrm{~mm}$ ( $\pm 16,25)$ (Tabla 1$)$.

Las hembras tuvieron LM entre 80 y $220 \mathrm{~mm} \mathrm{LM}$ durante la temporada 2002, y de 90 a 200 mm LM durante el 2003. La mayor abundancia correspondió a un grupo modal con un intervalo de 120 a 130 mm LM para ambas temporadas de pesca (Fig. 2a, b). En tanto que para los machos, el intervalo varió de 90 a 170 mm de longitud de manto y un pico de abundancia en 120 a $130 \mathrm{~mm}$ de LM en 2002 y de 110 a 180 mm de longitud de manto en 2003, con la mayor abundancia en el intervalo 130-140 mm de LM (Fig. 2c, d). Es de resaltar que el número de machos fue bajo, y con peso promedio sobre los $700 \mathrm{~g}$. En el caso de las hembras el intervalo de LM fue de 120 a $130 \mathrm{~mm}$, con un número mayor de organismos (536) en la temporada 2003.
Tabla 1. Número de organismos y proporción por sexos en las temporadas de pesca en Holbox, Quintana Roo, México / Number of organisms and sex proportion during fishing seasons at Holbox, Quintana Roo, Mexico

\begin{tabular}{lrrrr}
\hline Temporada de pesca & Hembras & Machos & Total & $\begin{array}{c}\text { Proporción de sexo } \\
+: \hat{+}\end{array}$ \\
\hline 2002 & & & & \\
$\quad$ Agosto & 41 & 30 & 71 & $1,36: 1$ \\
Septiembre & 201 & 86 & 287 & $2,33: 1$ \\
Octubre & 102 & 65 & 167 & $1,56: 1$ \\
Total & 344 & 181 & 525 & $1,90: 1$ \\
2003 & & & & \\
Agosto & 70 & 12 & 82 & $5,83: 1$ \\
Septiembre & 27 & 2 & 29 & $13,5: 1$ \\
Octubre & 522 & 14 & 536 & $37,28: 1$ \\
Noviembre & 146 & 6 & 152 & $24,33: 1$ \\
Total & 765 & 34 & 799 & $22,50: 1$ \\
\hline
\end{tabular}

La relación longitud peso indicó una relación alométrica; $\mathrm{P}=0,026 \mathrm{~L}^{2,16},\left(\mathrm{R}^{2}=0,71\right)$ para las hembras en 2002 y de $P=0,0076 L^{2,45}\left(R^{2}=0,74\right)$ en 2003 (Fig. 3a, b). En el caso de los machos la relación longitud peso fue de $\mathrm{P}=0,015 \mathrm{~L}^{2,27}\left(\mathrm{R}^{2}=0,69\right)$ para 2002, y de $\mathrm{P}=0,038$ $L^{2,12}\left(R^{2}=0,49\right)$ para 2003 , donde $P$ es el peso húmedo en g y L la longitud del manto en mm (Fig. 3c, d).

En ambos sexos se observó que el valor de la constante b no fue significativamente distinta de 3 , (t-Student $=7,89$, $P<0,05$ ), por lo que el crecimiento es del tipo alométrico. Los resultados del análisis con el ELEFAN no se ajustaron bien a los datos, ya que los valores de $\mathrm{Rn}$, en la superficie de respuesta fueron bajos $(<0,3)$ y las combinaciones de $\mathrm{K} \mathrm{y} \mathrm{L}_{\infty}$ no se consideraron biológicamente factibles. Contrariamente, utilizando el método Shepherd, se tuvieron mejores ajustes a los datos, con valores altos de correlación en la superficie de respuesta $(1,0)$, por ello fueron los que se consideraron para este trabajo. Como se tuvieron diferencias entre la abundancia de hembras y machos, los parámetros se calcularon por separado. Para las hembras se obtuvo una tasa de crecimiento de $\mathrm{K}=0,68$ $\mathrm{mm}$ año ${ }^{-1} \mathrm{y}$ una longitud asintótica de $\mathrm{L}_{\infty}=215 \mathrm{~mm}$ con un tiempo teórico $\mathrm{t}_{\mathrm{o}}=0,0344$. Para los machos, $\mathrm{K}=0,97$, y $\mathrm{L}_{\infty}=240,4 \mathrm{~mm}$ de LM. Considerando los parámetros poblacionales, $\mathrm{K}, \mathrm{L}_{\infty}$, $\mathrm{t}_{\mathrm{o}}$ de las hembras y utilizando la curva de captura, la mortalidad total fue de $\mathrm{Z}=3,16$. La mortalidad natural resultó de $\mathrm{M}=1,49$, y por lo tanto la mortalidad por pesca fue de $F=1,67$. El grado de explotación del recurso fue de $\mathrm{E}=0,53$. 


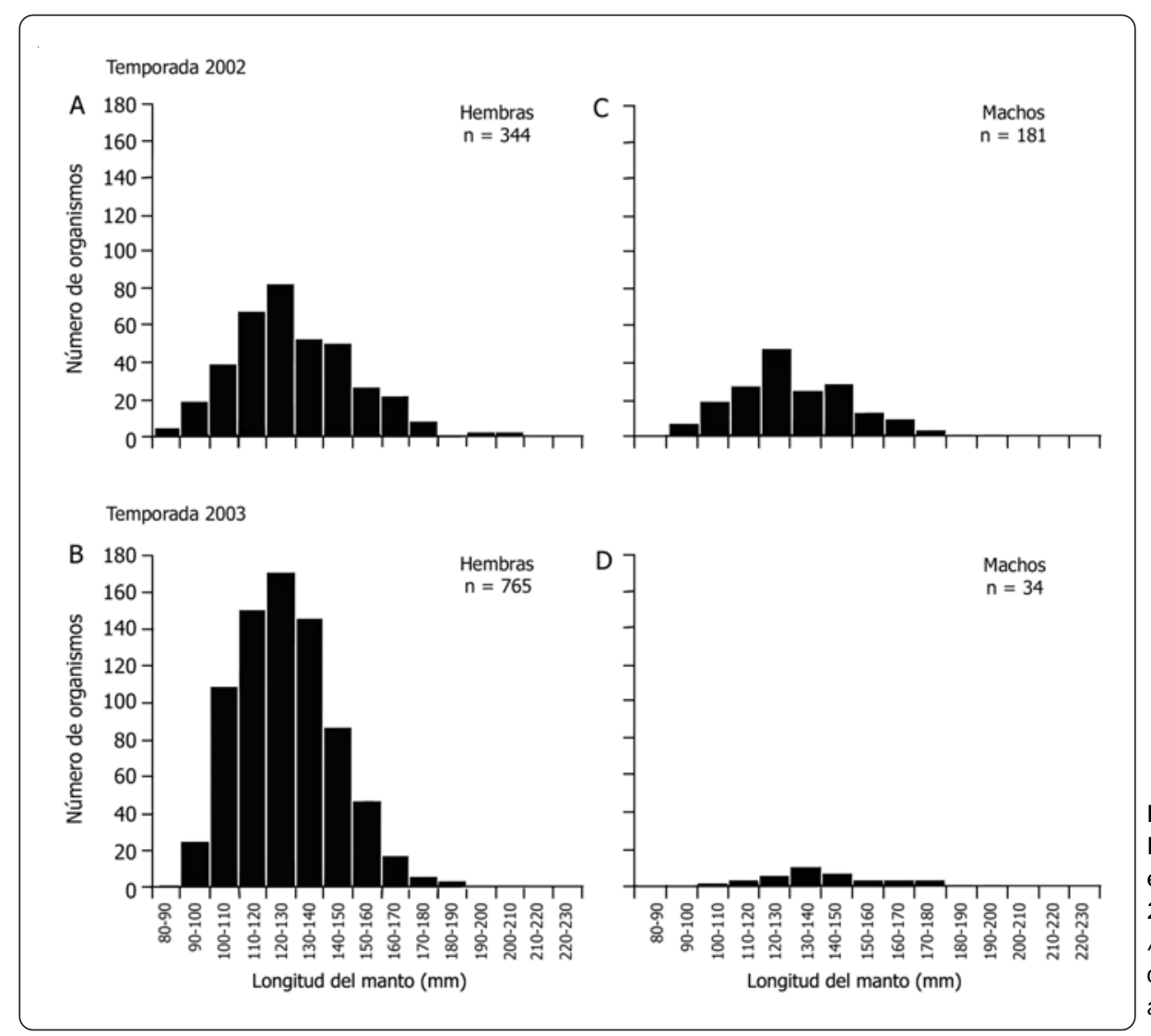

Figura 2. Histogramas de frecuencia longitud del manto de Octopus maya en las temporadas de pesca 2002 y 2003 Holbox, Quintana Roo, México / Mantle length frequency histograms of Octopus maya in fishing seasons 2002 and 2003, Holbox Quintana Roo, Mexico

\section{Discusión}

Las estimaciones de los valores de crecimiento y mortalidad de las poblaciones explotadas, constituyen parámetros de indudable importancia en la construcción de modelos para diseñar estrategias de manejo de los recursos pesqueros (Wright et al. 2009). Los análisis de los datos de frecuencia y longitud corporal son métodos indirectos comúnmente utilizados en la determinación de los parámetros poblacionales de los recursos biológicos. Esos datos deben cubrir preferentemente todo el intervalo de tamaños corporales de la especie en estudio, de tal manera que todos los grupos modales sean representados (Seijo et al. 1987). En este trabajo, el tamaño de la muestra fue adecuado, ya que proviene de la captura de la flota menor de Holbox y al muestrear la mayor cantidad de desembarques, se obtuvo una muestra proporcional a la captura, sin embargo, hay un sesgo en la información pues los organismos menores a la talla legal de captura, no estuvieron bien representados, por lo que se debe continuar el monitoreo, procurando una muestra de mayor tamaño y que incluya de preferencia a todos los estadios de la especie, en muestreos independientes de la pesquería.
En la composición de la población existió una diferencia significativa entre la proporción de machos y hembras, y esto puede deberse a una variedad de factores como: el agrupamiento natural por talla y sexo, el comportamiento, las diferentes tasas de crecimiento entre sexos, el desove y la mortalidad post desove (Rodríguez-Rúa et al. 2005), en este caso, no tenemos una explicación sólida para la mayor abundancia de hembras, por lo que se deberá iniciar una investigación para conocer la poca abundancia de machos en la población. Se ha mencionado que las hembras del pulpo $O$. vulgaris crecen más lento que los machos, quienes alcanzan la madurez sexual en la fase de crecimiento rápido, lo que puede representar una estrategia reproductiva de la especie (Boyle \& Rodhouse 2005), mientras que en las hembras, la maduración sexual se alcanza casi al final de la vida (Forsythe \& Van Heukelem 1987).

La relación longitud peso resultó ser alométrico, y esto coincide con lo que se ha documentado para el pulpo $O$. hubbsorum en el Pacífico mexicano (Alejo-Plata et al. 


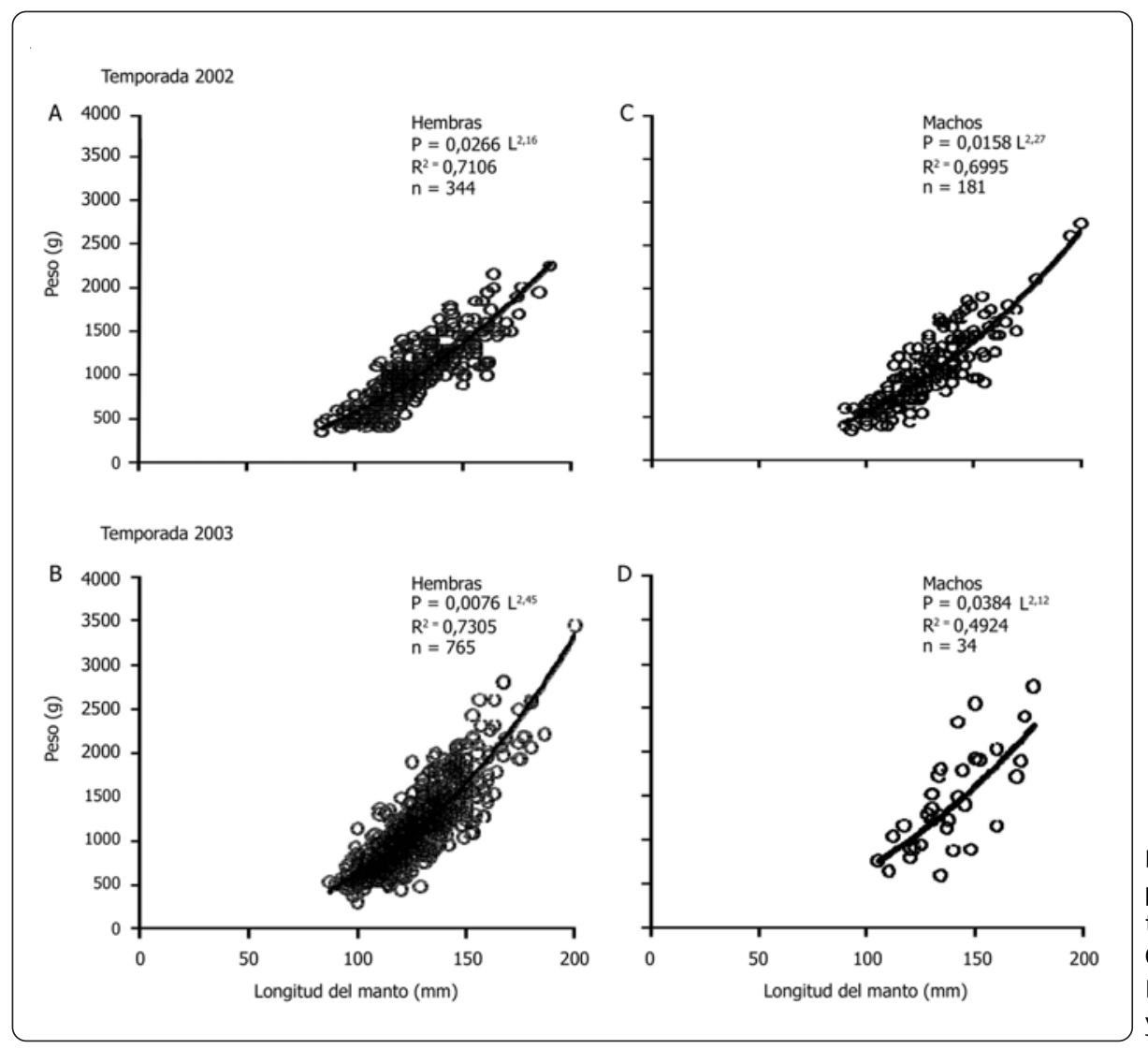

Figura 3. Relación longitud-peso del pulpo Octopus maya en las dos temporadas de pesca en Holbox, Quintana Roo, México / Octopus maya length-weight relationship in two fishing years at Holbox, Quintana Roo, Mexico

2009) y en Perú, donde Villegas \& Tafur (2000) reportaron un crecimiento alométrico para el pulpo Octopus mimus, con los siguientes parámetros: $a=0,0019, b=2,65$ para hembras y $a=0,0011, b=2,79$ para machos. En el caso de los invertebrados de tejido suave, como el pulpo, se ha mencionado que el análisis de los datos de frecuencialongitud no representan adecuadamente la talla del organismo, ya que éste puede tener distintos grados de contracción muscular (Forsythe \& Van Heukelem 1987), no obstante, estos métodos tienen la ventaja de permitir realizar evaluaciones rápidas de un recurso pesquero a bajo costo (Semmens et al. 2004), pero los resultados debieran ser validados con otras técnicas como marcaje-recaptura (Domain et al. 2000) o bien técnicas moleculares (Moltschaniwskyj 2004). Los parámetros de crecimiento obtenidos en este estudio: $\mathrm{L}_{\infty}=215 \mathrm{~mm}$ de $\mathrm{LM}$ y $\mathrm{K}=0$,68 año ${ }^{-1}$, son ligeramente menores a los encontrados en otras investigaciones realizadas en la Península de Yucatán. Arreguín-Sánchez et al. (2000) estimaron una $\mathrm{L}_{\infty}$ de 252 mm con una constante de crecimiento de $1,4 \mathrm{año}^{-1} \mathrm{y}$ el parámetro de ajuste $t_{0}=0,82$.
Nepita-Villanueva \& Defeo (2001) encontraron con el método ELEFAN, una $\mathrm{L}_{\infty}$ entre 213 y $285 \mathrm{~mm}$ de longitud de manto y una $\mathrm{K}$ entre 0,50 y 1,35 por año ${ }^{-1}$. Aunque el método utilizado entre estudios no fue el mismo, si reflejan una similitud con las poblaciones de Holbox. En general, se acepta que la especie tiene un ciclo de vida corto y una longevidad de 12 meses en cautiverio, y esto se refleja en la forma de crecimiento de la especie; con un crecimiento rápido, pero con alta variabilidad individual (Semmens et al. 2004, Storero et al. 2010). Algunos autores han modelado el crecimiento de los cefalópodos con diferentes expresiones matemáticas, y han concluido que el crecimiento no es asintótico (Alford \& Jackson 1993, Jackson et al. 2000), y aunque eso es contrario en los modelos que se ajustan a la curva de von Bertalanffy (Arreguín-Sánchez 1992a, Wolff \& Pérez 1992, NepitaVillanueva \& Defeo 2001), indicaría que no hay un modelo único que se adapte a la plasticidad del crecimiento de las diferentes especies de pulpo, ya que numerosas variables bióticas y abióticas pueden afectar el crecimiento individual (Semmens et al. 2004, Storero et al. 2010). En el caso de recursos pesqueros en regiones con poca 
investigación, la información generada aun con métodos indirectos, podría ser muy útil para proponer medidas de manejo del recurso, como en el caso de Holbox.

Los valores de mortalidad natural y por pesca $(\mathrm{M}=$ $1,49, Z=3,16)$ obtenidos en este estudio son similares a los encontrados previamente en la zona. Arreguín-Sánchez et al. (2000) estimaron una mortalidad natural $\mathrm{M}=2,2 \mathrm{y}$ una mortalidad total de 8,77, valores altos comparados con los estimados por Chávez (1998) en el Banco de Campeche $(Z=0,678)$ y los obtenidos en el presente estudio. Arreguín-Sánchez et al. (2000) concluyeron que el recurso en Yucatán estaba sometido a una intensa explotación, ya que $\mathrm{F} / \mathrm{Z}$ fue de 0,75 , con capturas realizadas por una flota mayor. En el caso de la pesca de pulpo en Holbox, la tasa de explotación (E) fue de 0,53 , y de acuerdo con lo establecido por Gulland (1971), el óptimo de explotación de un recurso ocurre cuando $E$ es cercano a 0,5 . Por lo tanto, se podría afirmar que la pesquería del pulpo en Holbox se encuentra cercana a un nivel óptimo. Las actividades de la flota artesanal en esta zona, aún no han mostrado efectos negativos comprobados sobre la población del pulpo, por lo que, sería recomendable mantener el esfuerzo de pesca, y continuar con los monitoreos en beneficio del recurso. Este trabajo es una primera aproximación a la pesquería de pulpo en Quintana Roo. Es importante que investigaciones futuras continuen con métodos más integrados, para así asegurar la sustentabilidad del recurso.

\section{Agradecimientos}

AHS, a El Colegio de la Frontera Sur, Unidad Chetumal por la oportunidad de mi desempeño como tesista. Al Consejo Quintanarroense de Ciencia y Tecnología del Estado de Quintana Roo, por el apoyo Económico Complementario Para Tesis. A Don Beto Bolas por todas las facilidades brindadas en la Isla de Holbox, y a Don Nico permisionario de pulpo, y quien facilitó el acceso de datos. A todos los pescadores de la cooperativa Isla Holbox y a la cooperativa Vanguardia del Mar. A Juan Carlos Pérez por la revisión crítica del manuscrito.

\section{LITERATURA CITADA}

Alejo-Plata MC, JL Gómez-Márquez, S Ramos-Carrillo \& JE Herrera-Galindo. 2009. Reproducción, dieta y pesquería del pulpo Octopus (Octopus) hubbsorum (Mollusca: Cephalopoda) en la costa de Oaxaca, México. Revista de Biología Tropical 57(1-2): 63-78.
Alford RA \& GD Jackson. 1993. Do cephalopods and larvae of other taxa grow asymptotically? The American Naturalist 141: 717-728.

Arreguín-Sánchez F. 1992a. Consideraciones sobre el manejo de la pesquería del pulpo Octopus maya en el banco de Campeche, México. Jaina 3: 1-18.

Arreguín-Sánchez F. 1992b. Growth and seasonal recruitment of Octopus maya on Campeche Bank, Mexico. Naga 15: 31-34.

Arreguín-Sánchez FJ, JC Seijo, D Fuentes \& M SolísRamírez. 1987. Estado de conocimiento de los recursos pesqueros de la Plataforma de Yucatán y región adyacente. Contribuciones de Investigación Pesquera. Documento técnico 4: 1-41. Secretaria de Pesca, Instituto Nacional de la Pesca, Centro Regional de Investigaciones Pesquera, Yucaltepén.

Arreguín-Sánchez F, M Solís-Ramírez \& ME González de la Rosa. 2000. Population dynamic and stock assessment for Octopus maya (Cephalopoda: Octopodidae) fishery in the Campeche Bank, Gulf of México. Revista de Biología Tropical 48(2-3): 323-331.

Boyle PR \& P Rodhouse. 2005. Cephalopods. Ecology and fisheries, $452 \mathrm{pp}$. Blackwell, Oxford.

Chávez EA. 1998. Estrategias óptimas de explotación del pulpo de la Sonda de Campeche México. Hidrobiológica 8(2): 97-105.

Defeo O, F Arreguín-Sánchez \& J Sánchez. 1992. Growth study for the yellow clam Mesodesma mactroides: a comparative analysis of three length based methods. Scientia Marina 56: 53-59.

De la Peña ME. 1957. Estudios anatómicos acerca del pulpo (Octopus vulgaris Lam) Tesis profesional, Facultad de Ciencias, Universidad Nacional Autónoma de México, México, 56 pp.

Domain F, D Jouffre \& A Cavarivière. 2000. Growth of Octopus vulgaris from tagging in Senegalese waters. Journal of the Marine Biological Association of the United Kingdom 80: 699-706.

Forsythe W \& WF Van Heukelem. 1987. Growth. En: Boyle PR (ed). Cephalopods life cycles. 2. Comparative reviews, pp. 135-156. Academic Press, London.

Fuentes DM, M Solís-Ramírez \& JM de la Garza. 1965. Algunos aspectos de la reproducción del pulpo Octopus vulgaris Lamarck de la Sonda de Campeche, 9 pp. Secretaria de Industria y Comercio, Dirección General de Industrias Conexas, México.

Gayanilo FC Jr, M Soriano \& D Pauly. 1989. A draft guide to the complete ELEFAN. ICLARM Software 2, $65 \mathrm{pp}$. International Center for Living Aquatic Resources Management, Manila. 
Gulland JA. 1971. Science and fishery management. Journal du Conseil, Conseil International pour l'Exploration de la Mer 33: 471-477.

Jackson GD, RA Alford \& JH Choat. 2000. Can length frequency analysis be used to determine squid growth? An assessment of ELEFAN. ICES Journal of Marine Science 57: 948-954.

Moltschaniwskyj NA. 2004. Understanding the process of growth in cephalopods. Marine and Freshwater Research 55: 379-386.

Nepita-Villanueva MR \& O Defeo. 2001. Crecimiento del pulpo Octopus maya (Mollusca: Cephalopoda) de la costa de Yucatán, México: un análisis de largo plazo. Revista de Biología Tropical 49: 93-101.

Pauly D. 1987. A review of the ELEFAN system for analysis of length frequency data in fish and aquatic invertebrates. En: Pauly D \& GP Morgan (eds). Length based methods in fisheries research. ICLARM Conference Proceedings 13: 7-31.

Rodríguez-Rúa A, I Pozuelo \& MA Prado. 2005. The gametogenic cycle of Octopus vulgaris (Mollusca: Cephalopoda) as observed on the Atlantic coast of Andalucía (south of Spain). Marine Biology 147: 927-933.

Seijo JC, MJ Solís-Ramírez \& G Morales. 1987. Simulación bioeconómica de la pesquería de pulpo Octopus maya de la plataforma continental de Yucatán. Memorias del Simposio Investigación Biología y Oceanografía Pesquera, México. La Paz, B.C.S., Abril 1987, pp. 125-138.

Semmens, JM, GT Pecl, R Villanueva, D Jouffre, I Sobrino, JB Wood \& PR Rigby. 2004. Understanding octopus growth: patterns, variability and physiology. Marine and Freshwater Research 55: 367-377.

Solís-Ramírez MJ. 1962. Contribución al estudio del pulpo (Octopus vulgaris Lamarck) de la Sonda de Campeche. Trabajos de Divulgación 3(24): 1-30.

Solís-Ramírez MJ. 1967. Aspectos biológicos del pulpo Octopus maya Voss y Solís. Publicaciones del Instituto Nacional de Investigaciones Pesqueras, México 18: 1-190.

Solís-Ramírez MJ. 1975. Posibilidades de la pesca de pulpo de la Península de Yucatán. Publicación, Instituto Mexicano de Comercio Exterior 347: 1-20.
Solís-Ramírez MJ. 1988. El recurso pulpo del Golfo de México y del Caribe Mexicano. En: SEPESCA (ed). Los recursos pesqueros del país. XXV Aniversario del INP, pp. 463478. Secretaría de Pesca y Agricultura, México.

Solís-Ramírez MJ \& EA Chávez. 1986. Evaluación y régimen óptimo de pesca del pulpo en la Península de Yucatán. Anales del Instituto de Ciencias del Mar y Limnología, UNAM 13: 1-18.

Solís Ramírez MJ, F Arreguín-Sánchez \& JC Seijo. 1999. Pesquerías de cefalópodos. En: Flores-Hernández D, P Sánchez-Gil, JC Seijo \& F Arreguín-Sánchez (eds). Análisis y diagnóstico de los recursos pesqueros críticos del Golfo de México, EPOMEX Serie Científica 7: 61-80. Universidad Autónoma de Campeche, Campeche.

Sosa-Cordero E, A Ramírez-González, M Domínguez \& A Aguilar-Perera. 1991. Peces y pesquerías del sur de Quintana Roo. Zona marítima. En: Camarena-Luhrs T \& SI Salazar-Vallejo (eds). Estudios ecológicos preliminares del sur de Quintana Roo, pp. 135-166. Centro de Investigaciones de Quintana Roo, Chetumal

Storero LP, M Ocampo-Reinaldo, R González \& MA Narvarte. 2010. Growth and life span of the small octopus Octopus tehuelchus in San Matías Gulf (Patagonia): three decades of study. Marine Biology 157: 555-564.

Villegas P \& R Tafur. 2000. Aspectos reproductivos del pulpo (Octopus mimus) en el área de Callao. Informes Progresivos, Instituto del Mar de Perú 121: 3-15.

Wright-López H, O Holguín-Quiñones, F Arreguín-Sánchez \& I Roque-Villada. 2009. Crecimiento y mortalidad de la madreperla Pinctada mazatlanica en poblaciones naturales del litoral oriental de Baja California Sur, México. Revista de Biología Tropical 57(1-2): 53-61

Wolff M \& H Pérez. 1992. Population dynamics, food consumption and gross conversion efficiency of Octopus mimus Gould, from Antofagasta (northern Chile). ICES Council Meeting Papers 1992: 1-12. [ICES-Shellfish Commitee C.M, Copenhagen Denmark]

Zar JH. 1999. Biostatistical analysis, 663 pp. Prentice Hall, Englewood Cliffs.

Recibido el 11 de mayo de 2010 y aceptado el 17 de agosto de 2010 